\title{
Anastomotic Leak Does Not Impact on Long-Term Outcomes in Esophageal Cancer Patients
}

\author{
S. K. Kamarajah, BMedSci, MBChB ${ }^{1,2}$, M. Navidi, MB, ChB, FRCSEd ${ }^{1}$, S. Wahed, MD, FRCS ${ }^{1}$, \\ A. Immanuel, MD, FRCSEd ${ }^{1}$, N. Hayes, FRCS $^{1}$, S. M. Griffin, OBE, MD, PRCSEd ${ }^{1}$, and \\ A. W. Phillips, MD, MA, FRCSEd ${ }^{1,3}$ \\ ${ }^{1}$ Northern Oesophagogastric Unit, Royal Victoria Infirmary, Newcastle University Trust Hospitals, Newcastle upon Tyne, \\ UK; ${ }^{2}$ Institute of Cellular Medicine, Newcastle University, Newcastle upon Tyne, UK; ${ }^{3}$ School of Medical Education, \\ Newcastle University, Newcastle upon Tyne, UK
}

\begin{abstract}
Background. Esophagectomy is a technically demanding procedure associated with high levels of morbidity. Anastomotic leak (AL) is a common complication with potentially major ramifications for patients. It has also been associated with poorer long-term overall survival (OS) and disease recurrence.

Objective. The aim of this study was to determine whether $\mathrm{AL}$ contributes to poor OS and recurrence-free survival (RFS) for patients with esophageal cancer.

Methods. Consecutive patients undergoing a two-stage, two-field transthoracic esophagectomy from a single highvolume unit between 1997 and 2016 were evaluated. Clinicopathologic characteristics, along with oncological and postoperative outcomes, were stratified by no $\mathrm{AL}$ versus non-severe leak (NSL) versus severe esophageal $\mathrm{AL}$ (SEAL). SEAL was defined as ALs associated with Clavien-Dindo grade III/IV complications.

Results. This study included 1063 patients, of whom 8\% (87/1063) developed AL; $45 \%$ of those who developed AL were SEALs (39/87). SEAL was associated with a prolonged critical care stay (median 8 vs. 3 vs. 2 days; $p<0.001$ ) and prolonged hospital stay (median 43 vs. 27 vs. 15 days; $p<0.001$ ) compared with NSL or no AL. There were no significant differences in number of lymph nodes harvested and rates of $\mathrm{R} 1$ resection between groups.
\end{abstract}

(C) The Author(s) 2020

First Received: 1 September 2019; Published Online: 23 January 2020

A. W. Phillips, MD, MA, FRCSEd

e-mail: awphillips@doctors.net.uk
OS and RFS were not affected by either NSL or SEAL, and Cox multivariate regression showed NSL and SEAL were not independently associated with OS and RFS. Sensitivity analysis in patients receiving neoadjuvant therapy followed by esophagectomy demonstrated similar findings.

Conclusion. These results demonstrate that AL leads to prolonged critical care and in-hospital length of stay; however, contrary to previous reports, our results do not compromise long-term outcomes and are unlikely to have a detrimental oncological impact.

Esophagectomy remains a key component of treatment for patients with potentially curable esophageal cancer. While mortality levels from the procedure have fallen dramatically over the last 30 years, esophagectomy is still associated with high levels of morbidity. ${ }^{1-3}$ Anastomotic leak (AL) is a commonly seen complication that has historically been associated with high mortality rates. ${ }^{4}$ The Esophagectomy Complications Consensus Group (ECCG) defined $\mathrm{AL}$ as a full-thickness defect involving the esophagus, anastomosis, staple line or conduit, irrespective of the presentation or method of identification. ${ }^{5}$ The classification further divided leaks into the management strategy employed: type I, those that require no change in treatment; type II, leaks that require intervention, but not surgery; and type III, leaks that require surgical intervention.

The incidence of AL has been reported at between 3 and $30 \% .{ }^{6,7}$ This can result in a prolonged hospital stay, a need for reoperation, anastomotic stricturing that requires repeated dilations, and potentially poorer long-term survival. ${ }^{8,9}$ A French multicenter study, which defined severe 
esophageal leaks (SEALs) as those that equated to a grade III/IV Clavien-Dindo complication, demonstrated poor long-term prognosis in patients who developed SEAL following esophagectomy. ${ }^{9}$ This study was limited by variations in oncological and surgical pathways and included a mixture of high- and low-volume units. Furthermore, this study did not report on recurrence-free survival (RFS).

Despite the above study, the impact of AL is unclear, with conflicting evidence, and the majority of the published literature are limited by small series. ${ }^{4,9-12}$ The aim of this study was to evaluate outcomes from a single high-volume center and to determine whether AL impacts on oncological and postoperative outcomes as well as long-term overall survival (OS) and RFS.

\section{METHODS}

\section{Patient Population}

Consecutive patients from the Northern Oesophagogastric Unit, Newcastle upon Tyne, treated for adenocarcinoma or squamous cell carcinoma (SCC) of the esophagus or gastroesophageal junction between January 1997 and December 2016 were included. All patients were discussed at a multidisciplinary meeting and subsequently received neoadjuvant chemo(radio)therapy followed by transthoracic esophagectomy (Ivor Lewis). Patients were identified from a contemporaneously maintained database.

\section{Pretreatment Staging}

All patients were staged according to standardized protocols, which included endoscopy with biopsy, endoscopic ultrasonography, external ultrasonography of the neck (if required), and a thoracoabdominal computed tomography (CT) scan. A positron emission tomography (CT) scan is used in patients being considered for radical (curative) treatment. In patients with histology proven, locally advanced resectable malignancy without metastases (cT1N + or cT3N0-3) or tumors of questionable resectability (cT4), neoadjuvant chemo(radio)therapy followed by surgery is the main treatment option. Patients with a histology other than adenocarcinoma or SCC and metastatic disease at the time of operation were excluded.

\section{Treatment}

Multiple neoadjuvant regimens were employed in the present study, determined by the standard of care and recruiting clinical trials at the time of treatment (Table 1), with patients treated earlier in the time period having unimodality surgery. The majority of patients treated received neoadjuvant chemotherapy. Transthoracic esophagectomy with two-field lymph node dissection was performed within 4-8 weeks after completion of the neoadjuvant therapy using a conventional or minimally invasive approach as previously described. ${ }^{13}$

\section{Pathology and Staging}

Histopathological reporting was carried out by specialist gastrointestinal pathologists using a standardized proforma. This was in line with guidelines produced by the Royal College of Pathologists, which included tumor type and differentiation, depth of tumor infiltration, and tumor regression. ${ }^{14}$ The total number of nodes from each location, as well as nodal metastases, were recorded, along with the presence of extracapsular, lymphatic, and venous and perineural invasion. Lymph nodes were dissected from the specimen by the operating surgeon and analyzed separately by the pathologist. ${ }^{15}$ The pathological stage was determined using the American Joint Committee on Cancer (AJCC) 8th edition TNM staging system. ${ }^{16}$

\section{Definition of Anastomotic Leak}

AL was defined as a full-thickness gastrointestinal defect involving the esophagus, anastomosis, staple line, or conduit irrespective of presentation or method of identification according to the ECCG criteria. Type I AL was defined as a local defect requiring no change in therapy, or treated medically or with dietary modification; type II AL was defined as a localized defect requiring interventional but not surgical therapy, for example interventional radiology drain, stent or bedside opening, and packing of incision; and type III AL was defined as a localized defect requiring surgical therapy. In this study, patients with $\mathrm{AL}$ associated with grade III-V complications, as defined by the Clavien-Dindo ${ }^{17}$ grading system, were defined as severe esophageal AL (SEAL), and those with less severe complications (Clavien-Dindo grade I/II were classified as non-severe leaks [NSLs]). Postoperative contrast swallows were not routinely used to determine if a leak was present and investigations were performed if there was clinical concern.

\section{Follow-Up and Definition of Recurrence}

Patients were followed up until death or for 10 years. Patients were seen at 3- to 6-monthly intervals in the first 2 years, 6-monthly for 2 years, and then annually. Recurrence of disease was based on clinical grounds and was confirmed endoscopically or radiologically. The minimum follow-up was 30 months. 
TABLE 1 Clinicopathologic characteristics of patients undergoing esophagectomy for esophageal cancer

\begin{tabular}{|c|c|c|c|c|c|}
\hline & Overall $[n=1063]$ & No AL $[n=976]$ & $\mathrm{AL}[n=48]$ & SEAL $[n=39]$ & $p$ value \\
\hline Age at presentation, years & $65(58-71)$ & $65(58-71)$ & $64(60-71)$ & $66(60-72)$ & 0.700 \\
\hline Sex, male & $811(76)$ & 739 (76) & $41(85)$ & $31(79)$ & 0.271 \\
\hline Histology, SCC & 207 (19) & $190(19)$ & $12(25)$ & $5(13)$ & 0.361 \\
\hline BMI, $\mathrm{kg} / \mathrm{m}^{2}$ & $26(24-29)$ & $26(24-29)$ & $27(23-30)$ & $26(24-29)$ & 0.777 \\
\hline Smoking status & & & & & 0.302 \\
\hline Current & $260(24)$ & $233(24)$ & $17(35)$ & $10(26)$ & \\
\hline Ex-smoker & $493(46)$ & $449(46)$ & $23(48)$ & $21(54)$ & \\
\hline Never & $302(28)$ & $286(29)$ & $8(17)$ & $8(21)$ & \\
\hline Unknown & $8(1)$ & $8(1)$ & $0(0)$ & $0(0)$ & \\
\hline Alcohol status & & & & & 0.009 \\
\hline Current & $770(72)$ & $703(72)$ & $34(71)$ & $33(85)$ & \\
\hline Ex-drinker & $75(7)$ & $63(6)$ & $9(19)$ & $3(8)$ & \\
\hline Never & 199 (19) & $191(20)$ & $5(10)$ & $3(8)$ & \\
\hline Unknown & $19(2)$ & $19(2)$ & $0(0)$ & $0(0)$ & \\
\hline ASA grade & & & & & 0.309 \\
\hline 1 & $158(15)$ & $145(15)$ & $9(19)$ & $4(10)$ & \\
\hline 2 & $539(51)$ & $496(51)$ & $19(40)$ & $24(62)$ & \\
\hline 3 & $270(25)$ & $247(25)$ & $16(33)$ & $7(18)$ & \\
\hline 4 & $7(1)$ & $5(1)$ & $1(2)$ & $1(3)$ & \\
\hline Unknown & $89(8)$ & $83(9)$ & $3(6)$ & $3(8)$ & \\
\hline Overall treatment, surgery only & $500(47)$ & $458(47)$ & $17(35)$ & $25(64)$ & 0.028 \\
\hline Neoadjuvant chemotherapy regimen & & & & & 0.035 \\
\hline $\mathrm{CF}$ & $208(20)$ & $184(19)$ & $17(35)$ & $7(18)$ & \\
\hline CROSS & $20(2)$ & $18(2)$ & $2(4)$ & $0(0)$ & \\
\hline $\mathrm{ECF} / \mathrm{ECX}$ & $285(27)$ & $267(27)$ & $11(23)$ & $7(18)$ & \\
\hline None & $500(47)$ & $458(47)$ & $17(35)$ & $25(64)$ & \\
\hline Unknown & $50(5)$ & $49(5)$ & $1(2)$ & $0(0)$ & \\
\hline Overall AJCC 8th edition pathological stage & & & & & 0.311 \\
\hline 0 & $49(5)$ & $45(5)$ & $3(6)$ & $1(3)$ & \\
\hline I & $243(23)$ & $223(23)$ & $8(17)$ & $12(31)$ & \\
\hline II & $234(22)$ & $209(21)$ & $12(25)$ & $13(33)$ & \\
\hline III & $450(42)$ & $415(43)$ & $23(48)$ & $12(31)$ & \\
\hline IV & $87(8)$ & $84(9)$ & $2(4)$ & $1(3)$ & \\
\hline Tumor grade & & & & & 0.222 \\
\hline Well & $94(9)$ & $87(9)$ & $2(4)$ & $5(13)$ & \\
\hline Moderate & $512(48)$ & $475(49)$ & $18(38)$ & $19(49)$ & \\
\hline Poor & $393(37)$ & $353(36)$ & $26(54)$ & $14(36)$ & \\
\hline Unknown & $64(6)$ & $61(6)$ & $2(4)$ & $1(3)$ & \\
\hline Lymph nodes harvested & $30(23-39)$ & $30(23-39)$ & $30(22-39)$ & $26(20-37)$ & 0.335 \\
\hline Margin status, R1 & $26(2)$ & $23(2)$ & $1(2)$ & $2(5)$ & 0.539 \\
\hline Lymphatic involvement & $490(46)$ & $452(46)$ & $24(50)$ & $14(36)$ & 0.378 \\
\hline Venous involvement & $373(35)$ & 347 (36) & $19(40)$ & 7 (18) & 0.062 \\
\hline Perineural involvement & $476(45)$ & $442(45)$ & $23(48)$ & $11(28)$ & 0.099 \\
\hline Tumor regression grade & & & & & 0.502 \\
\hline 1 & $38(4)$ & $35(4)$ & $2(4)$ & $1(3)$ & \\
\hline 2 & $30(3)$ & $29(3)$ & $1(2)$ & $0(0)$ & \\
\hline 3 & $79(7)$ & $72(7)$ & $5(10)$ & $2(5)$ & \\
\hline 4 & $163(15)$ & $151(15)$ & $10(21)$ & $2(5)$ & \\
\hline
\end{tabular}


TABLE 1 continued

\begin{tabular}{|c|c|c|c|c|c|}
\hline & Overall $[n=1063$ ] & No AL $[n=976]$ & $\mathrm{AL}[n=48]$ & SEAL $[n=39]$ & $p$ value \\
\hline 5 & $44(4)$ & $40(4)$ & $3(6)$ & $1(3)$ & \\
\hline Unknown & $709(67)$ & $649(66)$ & $27(56)$ & $33(85)$ & \\
\hline Extracapsular spread & $179(17)$ & $162(17)$ & $10(21)$ & $7(18)$ & 0.733 \\
\hline Critical care stay, days & $2(1-5)$ & $2(1-5)$ & $3(2-8)$ & $8(3-18)$ & $<0.001$ \\
\hline Total hospital stay, days & $15(12-22)$ & $15(12-21)$ & $27(15-40)$ & $43(32-64)$ & $<0.001$ \\
\hline Overall complications & $709(67)$ & $622(64)$ & $48(100)$ & $39(100)$ & $<0.001$ \\
\hline Surgical site infection & $108(10)$ & $100(10)$ & $4(8)$ & $4(10)$ & 0.912 \\
\hline Pulmonary complications & $123(12)$ & $109(11)$ & $9(19)$ & $5(13)$ & 0.268 \\
\hline Cardiac complications & $73(7)$ & $65(7)$ & $5(10)$ & $3(8)$ & 0.591 \\
\hline Anastomotic leaks & $87(8)$ & $0(0)$ & $48(100)$ & $39(100)$ & \\
\hline In-hospital mortality & $38(4)$ & $34(3)$ & $1(2)$ & $3(8)$ & 0.324 \\
\hline 30-day mortality & $28(3)$ & $26(3)$ & $0(0)$ & $2(5)$ & 0.325 \\
\hline
\end{tabular}

$A L$ anastomotic leaks, ASA American Society of Anesthesiologists, $S E A L$ severe esophageal AL, $S C C$ squamous cell carcinoma, $B M I$ body mass index, AJCC American Joint Committee on Cancer, CF Cisplatin and 5-Fluorouracil, CROSS Carboplatin, Paclitaxel and Radiotherapy, ECF Epirubicin, Cisplatin, 5-Fluorouracil, ECX Epirubicin, Cisplatin, Capecitabine

\section{Statistical Analysis}

Categorical variables were compared using the Chisquare test; non-normally distributed data were analyzed using the Mann-Whitney $U$ test; survival was estimated using Kaplan-Meier survival curves and compared using the log-rank test; and multivariable analyses used Cox proportional hazards models. A subset analysis in patients receiving neoadjuvant therapy prior to esophagectomy were analyzed. A $p$ value $<0.05$ was considered to be statistically significant. Data analysis was performed using R Foundation Statistical software (R 3.2.2) with TableOne, ggplot2, Hmisc, Matchit and survival packages (R Foundation for Statistical Computing, Vienna, Austria) as previously described. ${ }^{18,19}$

\section{RESULTS}

\section{Overall Cohort}

Baseline Demographics This study included 1063 patients undergoing esophagectomy for esophageal cancer, of whom $563(53 \%)$ received neoadjuvant therapy. Clinicopathological variables are presented in Table 1 . The median age of the entire cohort was 65 years (interquartile range 58-71 years), $76 \%$ were male, and $19 \%$ had SCC. Of the 1063 patients, $8 \%$ developed ALs (87/1063), of whom 45\% (39/87) developed SEAL. Two patients who developed AL (2\%) died.

Across the groups, there were no significant differences in age, sex, and rates of SCC; however, those developing SEAL were more likely to receive unimodality surgery
(64\%) compared with those with no ALs (47\%) or NSL only (35\%) [ $p=0.028]$. There were no significant differences in the rates of advanced tumor stage, number of lymph nodes examined, and R1 margins.

Postoperative Outcomes Patients developing SEAL had a significantly longer length of stay in critical care (median 8 vs. 3 vs. 2 days; $p<0.001$ ) and overall hospital stay (median 43 vs. 27 vs. 15 days; $p<0.001$ ) compared with NSL or no AL. However, there were no significant differences in the rates of surgical site infections, cardiac complications, pulmonary complications, and in-hospital mortality across these groups.

Overall and Recurrence-Free Survival There was no significant difference in OS between the groups, with patients experiencing SEAL having a median survival of 61 months, compared with 55 months for patients with NSL and 41 months for patients with no AL only $(p=0.8)$ (Fig. 1a). On Cox multivariate regression, both NSL only (hazard ratio [HR] $0.91 ; p=0.6$ ) and SEAL (HR 1.24; $p=0.3$ ) were not independently associated with OS (Table 2). Patients developing SEAL had similar RFS as patients with NSL or no AL (Fig. 1b). On Cox multivariate regression, NSL (HR 0.86; $p=0.5$ ) or SEAL (HR 1.19; $p=0.6)$ was not independently associated with OS (Table 2).

\section{Neoadjuvant and Surgery}

Baseline Demographics In this subgroup analysis, 563 patients were included. Clinicopathological variables are 

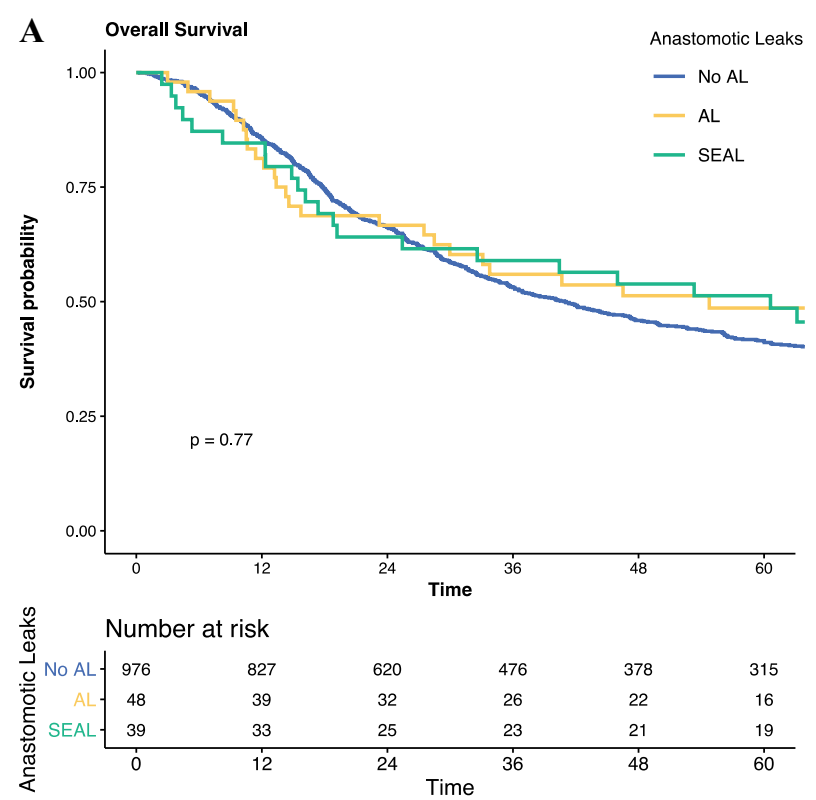

C
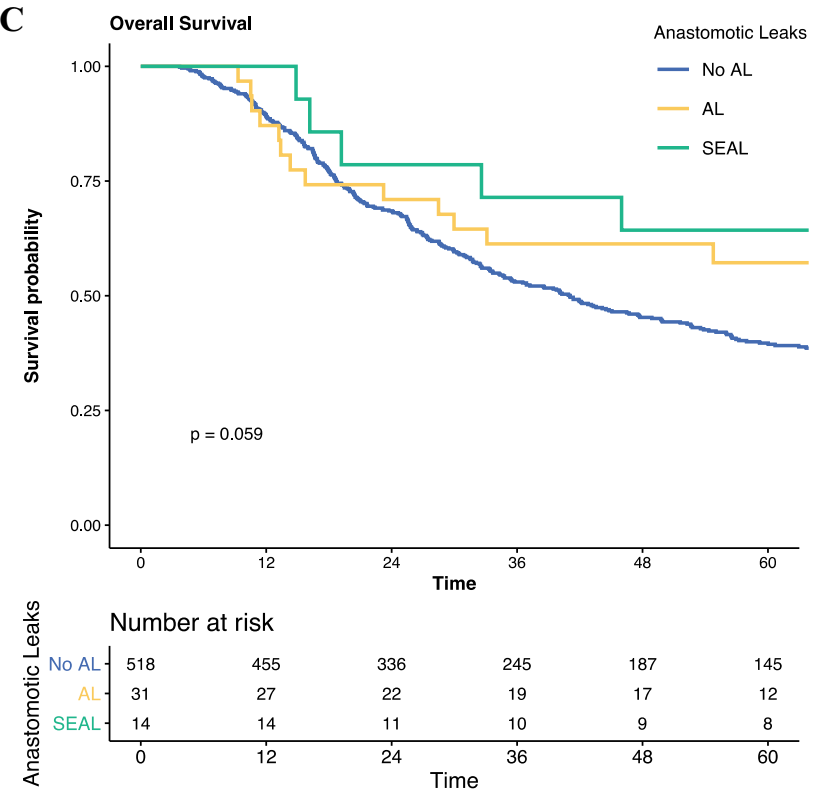

FIG. 1 Impact of SEAL on OS and RFS in all patients and neoadjuvant therapy and surgery only. a OS in all patients; b RFS in all patients; $\mathbf{c}$ OS in NAT and surgery and d RFS in NAT and

presented in Table 3. Of these patients, $8 \%$ developed ALs (45/563), of whom 31\% (14/45) developed SEAL. Across the groups, there were no significant differences in age, sex, and rates of SCC, and no significant differences in rates of advanced tumor stage, number of lymph nodes examined, and R1 margins.

Postoperative Outcomes Patients developing SEAL had significantly longer length of stay in critical care (median 8 vs. 4 vs. 2 days; $p<0.001$ ) and overall hospital stay
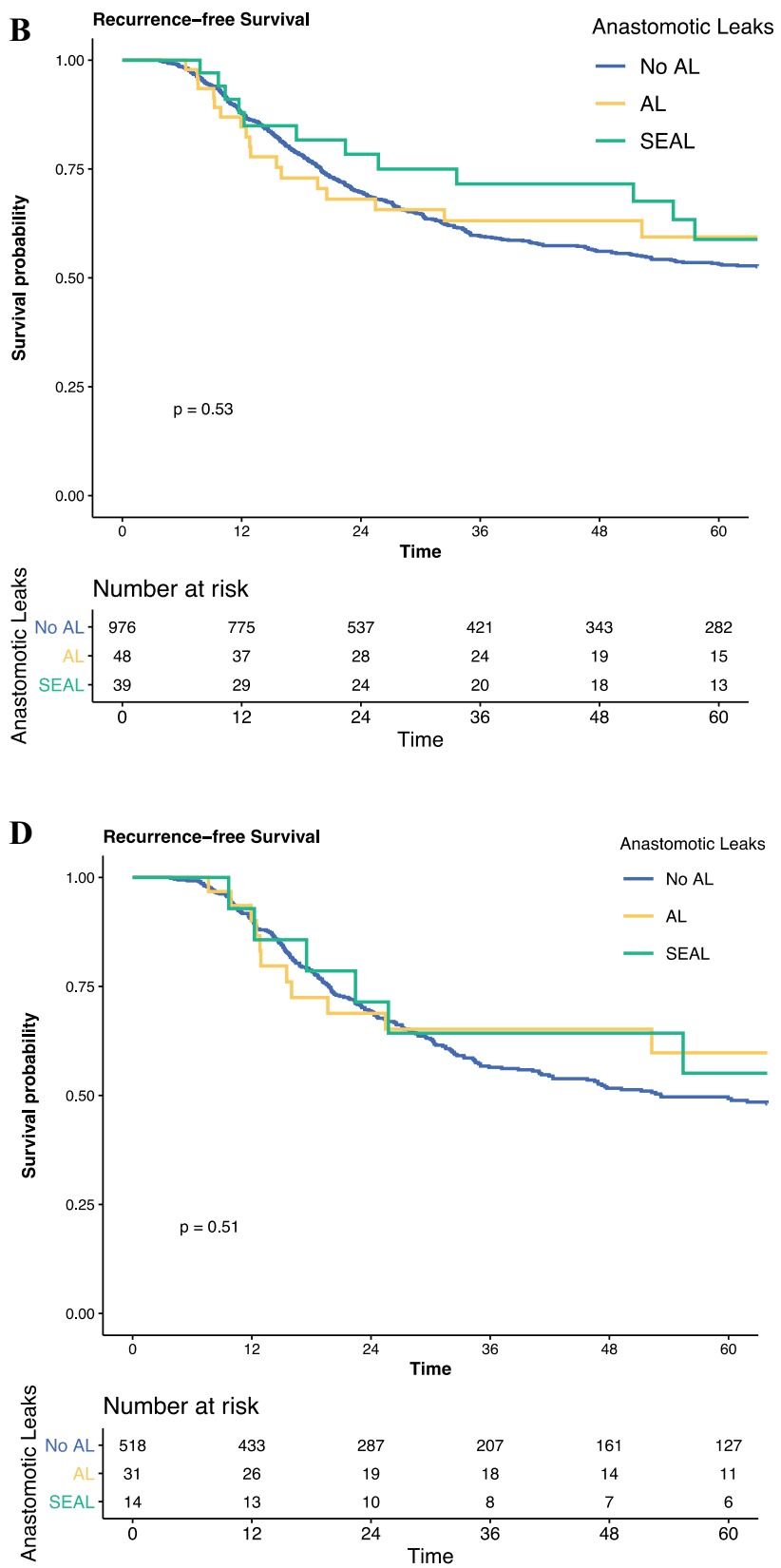

surgery. $A L$ anastomotic leak, $N R$ not reached, $N S L$ non-severe leak, $S E A L$ severe esophageal AL, OS overall survival, $R F S$ recurrence-free survival

(median 37 vs. 27 vs. 14 days; $p<0.001$ ) compared with NSL or no AL. However, there were no significant differences in the rates of surgical site infections, cardiac complications, pulmonary complications, and in-hospital mortality across these groups.

Overall and Recurrence-Free Survival There was no significant difference in survival between cohorts (SEAL: median, not reported [NR] vs. NSL: 77 vs. no leak: 41 months; $p=0.058$ ). On Cox multivariate regression, 
TABLE 2 Cox multivariate regression on the impact of SEAL on overall survival and recurrence-free survival

\begin{tabular}{|c|c|c|c|c|}
\hline & \multicolumn{2}{|l|}{ Overall survival } & \multicolumn{2}{|c|}{ Recurrence-free survival } \\
\hline & HR $(95 \% \mathrm{CI})$ & $p$ value & HR $(95 \% \mathrm{CI})$ & $p$ value \\
\hline Age at presentation & $1.02(1.01-1.03)$ & $<0.001$ & $1.00(0.99-1.01)$ & 0.991 \\
\hline Gender, male & $1.34(1.10-1.64)$ & 0.003 & $1.39(1.07-1.80)$ & 0.015 \\
\hline \multicolumn{5}{|l|}{ ASA grade } \\
\hline 1 & Ref & & Ref & \\
\hline 2 & $1.17(0.93-1.48)$ & 0.189 & $0.89(0.67-1.19)$ & 0.442 \\
\hline 3 & $1.30(1.01-1.68)$ & 0.041 & $0.99(0.72-1.35)$ & 0.93 \\
\hline 4 & $1.96(0.83-4.60)$ & 0.124 & $0.37(0.05-2.77)$ & 0.335 \\
\hline Unknown & $1.11(0.81-1.54)$ & 0.515 & $1.08(0.72-1.62)$ & 0.722 \\
\hline Histology, SCC & $1.30(1.04-1.61)$ & 0.019 & $1.10(0.82-1.48)$ & 0.522 \\
\hline Operation year & $0.96(0.94-0.98)$ & 0.001 & $0.97(0.94-1.00)$ & $\mathbf{0 . 0 3 8}$ \\
\hline Overall treatment, surgery only & $1.14(0.93-1.41)$ & 0.213 & $1.12(0.85-1.47)$ & 0.419 \\
\hline \multicolumn{5}{|l|}{ Tumor grade } \\
\hline Well & Ref & & Ref & \\
\hline Moderate & $1.28(0.92-1.78)$ & 0.141 & $2.22(1.21-4.08)$ & 0.01 \\
\hline Poor & $1.55(1.09-2.19)$ & 0.014 & $2.62(1.41-4.87)$ & 0.002 \\
\hline Unknown & $1.73(1.01-2.97)$ & 0.046 & $2.70(1.06-6.90)$ & $\mathbf{0 . 0 3 8}$ \\
\hline Margin status, R1 & $2.05(1.35-3.11)$ & 0.001 & $1.72(0.97-3.04)$ & 0.065 \\
\hline Lymphatic involvement, yes & $1.40(1.15-1.71)$ & 0.001 & $1.58(1.23-2.04)$ & $<0.001$ \\
\hline Venous involvement, yes & $1.00(0.83-1.22)$ & 0.971 & $1.07(0.84-1.36)$ & 0.59 \\
\hline Perineural involvement, yes & $1.42(1.17-1.72)$ & $<\mathbf{0 . 0 0 1}$ & $1.57(1.23-2.00)$ & $<\mathbf{0 . 0 0 1}$ \\
\hline \multicolumn{5}{|l|}{ Tumor regression grade } \\
\hline 1 & Ref & & Ref & \\
\hline 2 & $1.14(0.48-2.71)$ & 0.763 & $0.96(0.32-2.85)$ & 0.941 \\
\hline 3 & $0.94(0.45-1.95)$ & 0.871 & $0.74(0.30-1.83)$ & 0.52 \\
\hline 4 & $1.28(0.66-2.49)$ & 0.469 & $1.02(0.44-2.35)$ & 0.963 \\
\hline 5 & $1.35(0.65-2.79)$ & 0.42 & $0.99(0.40-2.47)$ & 0.984 \\
\hline Unknown & $1.25(0.66-2.36)$ & 0.498 & $0.82(0.36-1.83)$ & 0.625 \\
\hline Extracapsular spread, yes & $1.71(1.34-2.18)$ & $<\mathbf{0 . 0 0 1}$ & $1.42(1.06-1.91)$ & 0.02 \\
\hline \multicolumn{5}{|c|}{ Overall AJCC 8th edition pathological stage } \\
\hline 0 & Ref & & Ref & \\
\hline I & $1.09(0.59-2.00)$ & 0.788 & $1.94(0.58-6.41)$ & 0.279 \\
\hline II & $1.09(0.59-2.00)$ & 0.781 & $2.28(0.70-7.45)$ & 0.172 \\
\hline III & $2.40(1.31-4.40)$ & 0.005 & $5.93(1.82-19.28)$ & 0.003 \\
\hline IV & $3.57(1.85-6.88)$ & $<\mathbf{0 . 0 0 1}$ & $7.77(2.30-26.25)$ & 0.001 \\
\hline \multicolumn{5}{|l|}{ Anastomotic leaks } \\
\hline No anastomotic leaks & Ref & & Ref & \\
\hline NSL & $0.91(0.62-1.35)$ & 0.646 & $0.86(0.52-1.40)$ & 0.539 \\
\hline SEAL & $1.24(0.82-1.88)$ & 0.302 & $1.19(0.66-2.16)$ & 0.561 \\
\hline
\end{tabular}

ASA American Society of Anesthesiologists, SEAL severe esophageal anastomotic leak, SCC squamous cell carcinoma, AJCC American Joint Committee on Cancer, HR hazard ratio, CI confidence interval, Ref reference, $N S L$ non-severe leak

Bold values indicate statistical significance $(P<0.05)$ both NSL (HR 0.65; $p=0.1$ ) and SEAL (HR 0.48; $p=0.09$ ) were not independently associated with OS (Table 4). Patients developing SEAL had similar RFS as patients with NSL or no AL (Fig. 1d). On Cox multivariate regression, both NSL (HR $0.70 ; p=0.3$ ) or SEAL (HR $1.04 ; p=0.9)$ were not independently associated with OS (Table 4). 
TABLE 3 Clinicopathologic characteristics of patients undergoing esophagectomy following neoadjuvant therapy for esophageal cancer

\begin{tabular}{|c|c|c|c|c|}
\hline & No AL $[n=518]$ & $\operatorname{AL}[n=31]$ & SEAL $[n=14]$ & $p$ value \\
\hline Age at presentation, years & $64(57-69)$ & $63(60-70)$ & $66(60-73)$ & 0.581 \\
\hline Sex, male & 407 (79) & $26(84)$ & $13(93)$ & 0.346 \\
\hline Histology, SCC & $101(19)$ & $10(32)$ & $1(7)$ & 0.108 \\
\hline BMI, $\mathrm{kg} / \mathrm{m}^{2}$ & $26(24-30)$ & $28(25-30)$ & $26(24-29)$ & 0.702 \\
\hline Smoking status & & & & 0.169 \\
\hline Current & $121(23)$ & $14(45)$ & $4(29)$ & \\
\hline Ex-smoker & $246(47)$ & $11(35)$ & $8(57)$ & \\
\hline Never & $146(28)$ & $6(19)$ & $2(14)$ & \\
\hline Unknown & $5(1)$ & $0(0)$ & $0(0)$ & \\
\hline Alcohol status & & & & 0.274 \\
\hline Current & $391(75)$ & $24(77)$ & $12(86)$ & \\
\hline Ex-drinker & $31(6)$ & $4(13)$ & $2(14)$ & \\
\hline Never & $92(18)$ & $3(10)$ & $0(0)$ & \\
\hline Unknown & $4(1)$ & $0(0)$ & $0(0)$ & \\
\hline ASA grade & & & & 0.644 \\
\hline 1 & $74(14)$ & $6(19)$ & $1(7)$ & \\
\hline 2 & $292(56)$ & $12(39)$ & $9(64)$ & \\
\hline 3 & $124(24)$ & $12(39)$ & $3(21)$ & \\
\hline 4 & $2(0)$ & $0(0)$ & $0(0)$ & \\
\hline Unknown & $26(5)$ & $1(3)$ & $1(7)$ & \\
\hline Neoadjuvant chemotherapy regimen & & & & 0.179 \\
\hline $\mathrm{CF}$ & $184(36)$ & $17(55)$ & $7(50)$ & \\
\hline CROSS & $18(3)$ & $2(6)$ & $0(0)$ & \\
\hline $\mathrm{ECF} / \mathrm{ECX}$ & $267(52)$ & $11(35)$ & $7(50)$ & \\
\hline Unknown & $49(9)$ & $1(3)$ & $0(0)$ & \\
\hline Overall AJCC 8th edition pathological stage & & & & 0.683 \\
\hline 0 & $30(6)$ & $2(6)$ & $1(7)$ & \\
\hline I & $64(12)$ & $4(13)$ & $1(7)$ & \\
\hline II & $135(26)$ & $8(26)$ & $7(50)$ & \\
\hline III & $233(45)$ & $15(48)$ & $5(36)$ & \\
\hline IV & $56(11)$ & $2(6)$ & $0(0)$ & \\
\hline Tumor grade & & & & 0.768 \\
\hline Well & $17(3)$ & $1(3)$ & $1(7)$ & \\
\hline Moderate & $251(48)$ & $12(39)$ & $6(43)$ & \\
\hline Poor & $212(41)$ & $17(55)$ & $6(43)$ & \\
\hline Unknown & $38(7)$ & $1(3)$ & $1(7)$ & \\
\hline Lymph nodes harvested & $33(26-42)$ & $34(26-40)$ & $36(30-39)$ & 0.974 \\
\hline Margin status, R1 & $6(1)$ & $0(0)$ & $0(0)$ & 0.768 \\
\hline Lymphatic involvement & $259(50)$ & $15(48)$ & $6(43)$ & 0.86 \\
\hline Venous involvement & $190(37)$ & $11(35)$ & $2(14)$ & 0.227 \\
\hline Perineural involvement & $245(47)$ & $14(45)$ & $5(36)$ & 0.679 \\
\hline Tumor regression grade & & & & 0.822 \\
\hline 1 & $21(4)$ & $2(6)$ & $0(0)$ & \\
\hline 2 & $28(5)$ & $1(3)$ & $0(0)$ & \\
\hline 3 & $72(14)$ & $5(16)$ & $2(14)$ & \\
\hline 4 & $148(29)$ & $10(32)$ & $2(14)$ & \\
\hline 5 & $38(7)$ & $3(10)$ & $1(7)$ & \\
\hline
\end{tabular}


TABLE 3 continued

\begin{tabular}{|c|c|c|c|c|}
\hline & No AL $[n=518]$ & $\operatorname{AL}[n=31]$ & SEAL $[n=14]$ & $p$ value \\
\hline Unknown & $211(41)$ & $10(32)$ & $9(64)$ & \\
\hline Extracapsular spread & $127(25)$ & $9(29)$ & $4(29)$ & 0.809 \\
\hline Critical care stay, days & $2(1-4)$ & $4(2-8)$ & $8(2-16)$ & 0.001 \\
\hline Total hospital stay, days & $14(11-19)$ & $27(16-40)$ & $37(31-60)$ & $<0.001$ \\
\hline Overall complications & $316(61)$ & $31(100)$ & $14(100)$ & $<0.001$ \\
\hline Surgical site infection & $45(9)$ & $2(6)$ & $3(21)$ & 0.226 \\
\hline Pulmonary complications & $69(13)$ & $6(19)$ & $2(14)$ & 0.636 \\
\hline Cardiac complications & $48(9)$ & $5(16)$ & $1(7)$ & 0.43 \\
\hline Anastomotic leaks & $0(0)$ & $31(100)$ & $14(100)$ & $<0.001$ \\
\hline In-hospital mortality & $15(3)$ & $0(0)$ & $0(0)$ & 0.512 \\
\hline 30-day mortality & $10(2)$ & $0(0)$ & $0(0)$ & 0.643 \\
\hline
\end{tabular}

$A L$ anastomotic leak, $A S A$ American Society of Anesthesiologists, $H R$ hazard ratio, $S E A L$ severe esophageal AL, $S C C$ squamous cell carcinoma, $B M I$ body mass index, AJCC American Joint Committee on Cancer, $C F$ Cisplatin and 5-Fluorouracil, CROSS Carboplatin, Paclitaxel and Radiotherapy, ECF Epirubicin, Cisplatin, 5-Fluorouracil, ECX Epirubicin, Cisplatin, Capecitabine

\section{DISCUSSION}

The results of this study indicate that patients with AL, as well as those with severe ALs, do not have a poorer long-term survival than those patients who do not have an AL. In the short-term, NSL and SEAL were associated with a significantly longer stay in critical care and also longer time in hospital postoperatively. In-hospital mortality was not significantly different between those who had an $\mathrm{AL}$ and those who did not. In addition, there were comparable oncological outcomes between the groups in terms of R1 margin rates and number of lymph nodes harvested, which may reflect the similar survival between the groups, even after multivariable Cox regression analyses.

These findings are contrary to those of a previously published multicenter study that demonstrated that SEAL is associated with reduced long-term OS and worse recurrence rates. ${ }^{9}$ However, the previous study had several limitations that are imperative to understanding the impact of $\mathrm{AL}$ on survival. First, multicenter data are often heterogenous in regard to the type of surgery performed, which in this case included transhiatal and three-stage procedures that are established to have different survival profiles. In contrast, the present study focused only on patients who had undergone a two-stage transthoracic esophagectomy. Second, the previous multicenter study is limited by center variation in the context of volume, pathological assessment of specimens, and multidisciplinary pathways. ${ }^{9}$ Interestingly, center volume was associated with SEAL, which may also reflect the poor long-term survival outcomes. In addition, multicenter studies such as this, as well as other smaller studies, make it difficult to adjust for 30- and 90-day mortality. In the present study, all patients went through the same standardized multidisciplinary team process and were treated in a highvolume unit. There has been an increasing trend for esophagectomies to be carried out at high-volume units as this has been proven to improve both short- and long-term outcomes. ${ }^{20-22}$

The mechanism by which $\mathrm{AL}$ contributes towards poorer long-term survival is unclear. It has been postulated and extrapolated from colorectal surgery studies that cancer cells may be shed into the gut lumen during surgery, ${ }^{23}$ and thus anastomotic leakage allows these to spread into the mediastinum, contributing to local recurrence. ${ }^{9}$ There is conflicting evidence regarding whether perioperative morbidity impacts on long-term survival. While several studies have indicated that complications have no impact on survival, ${ }^{24,25}$ data from a Swedish national database suggested that surgical complications may be a poor predictor of long-term survival, ${ }^{26}$ and further studies from Japan have implicated pulmonary infections as having an unfavorable prognosis in patients who received neoadjuvant chemotherapy. ${ }^{10,27,28}$

In the present study, patients underwent a standardized two-field transthoracic esophagectomy, which has been previously described. Median lymph node yield was high and $\mathrm{R} 1$ resection rates low, both of which have been shown to contribute towards improved OS. ${ }^{29,30}$ There appears to be no difference in the impact of $\mathrm{AL}$, irrespective of whether or not a patient received neoadjuvant treatment. However, it may be that in the era of neoadjuvant treatment, the long-term oncological impact of an $\mathrm{AL}$ is lessened by this treatment. 
TABLE 4 Cox multivariate regression on the impact of SEAL on overall survival and recurrence-free survival

\begin{tabular}{|c|c|c|c|c|}
\hline & \multicolumn{2}{|l|}{ Overall survival } & \multicolumn{2}{|c|}{ Recurrence-free survival } \\
\hline & HR $(95 \% \mathrm{CI})$ & $p$ value & $\mathrm{HR}(95 \% \mathrm{CI})$ & $p$ value \\
\hline Age at presentation & $1.01(1.00-1.02)$ & 0.118 & $1.00(0.98-1.01)$ & 0.753 \\
\hline Gender, male & $1.41(1.04-1.92)$ & 0.027 & $1.21(0.84-1.73)$ & 0.306 \\
\hline \multicolumn{5}{|l|}{ ASA grade } \\
\hline 1 & Ref & & Ref & \\
\hline 2 & $0.95(0.69-1.32)$ & 0.77 & $0.90(0.61-1.32)$ & 0.581 \\
\hline 3 & $1.00(0.69-1.44)$ & 0.987 & $0.95(0.61-1.47)$ & 0.821 \\
\hline 4 & $0.95(0.21-4.37)$ & 0.952 & $0.53(0.07-4.35)$ & 0.557 \\
\hline Unknown & $0.80(0.45-1.42)$ & 0.448 & $0.82(0.41-1.64)$ & 0.578 \\
\hline Histology, SCC & $1.26(0.91-1.75)$ & 0.162 & $1.00(0.66-1.51)$ & 0.987 \\
\hline Operation year & $0.97(0.92-1.02)$ & 0.19 & $0.96(0.91-1.02)$ & 0.206 \\
\hline \multicolumn{5}{|l|}{ Tumor grade } \\
\hline Well & Ref & & Ref & \\
\hline Moderate & $1.35(0.68-2.68)$ & 0.392 & $1.19(0.54-2.59)$ & 0.668 \\
\hline Poor & $1.68(0.84-3.36)$ & 0.145 & $1.35(0.61-2.98)$ & 0.459 \\
\hline Unknown & $1.09(0.39-3.09)$ & 0.868 & $1.10(0.30-4.05)$ & 0.887 \\
\hline Margin status, R1 & $1.33(0.57-3.15)$ & 0.511 & $1.73(0.61-4.89)$ & 0.303 \\
\hline Lymphatic involvement, yes & $1.36(1.02-1.80)$ & 0.034 & $1.24(0.88-1.73)$ & 0.212 \\
\hline Venous involvement, yes & $1.00(0.76-1.31)$ & 0.999 & $1.06(0.77-1.47)$ & 0.708 \\
\hline Perineural involvement, yes & $1.51(1.15-1.97)$ & 0.003 & $1.81(1.30-2.51)$ & $<0.001$ \\
\hline \multicolumn{5}{|l|}{ Tumor regression grade } \\
\hline 1 & Ref & & Ref & \\
\hline 2 & $0.94(0.26-3.45)$ & 0.93 & $0.23(0.05-1.13)$ & 0.07 \\
\hline 3 & $0.84(0.26-2.76)$ & 0.779 & $0.30(0.08-1.21)$ & 0.09 \\
\hline 4 & $1.21(0.38-3.78)$ & 0.748 & $0.44(0.12-1.69)$ & 0.235 \\
\hline 5 & $1.33(0.41-4.35)$ & 0.637 & $0.45(0.11-1.79)$ & 0.255 \\
\hline Unknown & $1.11(0.35-3.53)$ & 0.863 & $0.32(0.08-1.23)$ & 0.097 \\
\hline Extracapsular spread, yes & $1.70(1.26-2.28)$ & $<\mathbf{0 . 0 0 1}$ & $1.40(0.99-1.99)$ & 0.057 \\
\hline \multicolumn{5}{|c|}{ Overall AJCC 8th edition pathological stage } \\
\hline 0 & Ref & & Ref & \\
\hline I & $0.74(0.27-2.08)$ & 0.572 & $3.98(0.76-20.94)$ & 0.103 \\
\hline II & $0.55(0.21-1.46)$ & 0.23 & $2.51(0.51-12.38)$ & 0.259 \\
\hline III & $1.43(0.53-3.85)$ & 0.474 & $7.01(1.37-35.80)$ & 0.019 \\
\hline IV & $1.74(0.61-4.98)$ & 0.301 & $7.98(1.49-42.91)$ & 0.015 \\
\hline \multicolumn{5}{|l|}{ Anastomotic leaks } \\
\hline No anastomotic leaks & Ref & & Ref & \\
\hline NSL & $0.65(0.39-1.09)$ & 0.105 & $0.70(0.38-1.30)$ & 0.259 \\
\hline SEAL & $0.48(0.21-1.12)$ & 0.09 & $1.04(0.45-2.43)$ & 0.919 \\
\hline
\end{tabular}

ASA American Society of Anesthesiologists, $H R$ hazard ratio, $C I$ confidence interval, $N S L$ non-severe leak, $R e f$ reference, SEAL severe esophageal anastomotic leak, SCC squamous cell carcinoma, AJCC American Joint Committee on Cancer

Bold values indicate statistical significance $(P<0.05)$
While it could be argued that one of the weaknesses of this study is that all the data come from a single unit, this is also one of its strengths as it provides evidence of potential outcomes for patients when looked after at an experienced center with a high volume of patients. The overall AL rate of $8 \%$ is comparable with other studies. ${ }^{4,10,15,26-29}$ For those patients who developed a leak, the mortality rate was low $(2 \%)$ and this translates to a $0.2 \%$ chance of dying from an $\mathrm{AL}$ after esophagectomy. While it is clear that $\mathrm{AL}$ 
is likely to prolong both critical care stay and the total time spent in hospital, an aggressive conservative management strategy can provide excellent outcomes. ${ }^{2}$

\section{CONCLUSION}

The present study refutes the suggestion that AL leads to poorer long-term oncological outcomes. A standardized esophagectomy with careful consideration for oncological principles and management of complications at a highvolume center can provide good short- and long-term outcomes after esophagectomy.

\section{FUNDING None declared.}

DISCLOSURE None declared.

OPEN ACCESS This article is licensed under a Creative Commons Attribution 4.0 International License, which permits use, sharing, adaptation, distribution and reproduction in any medium or format, as long as you give appropriate credit to the original author(s) and the source, provide a link to the Creative Commons licence, and indicate if changes were made. The images or other third party material in this article are included in the article's Creative Commons licence, unless indicated otherwise in a credit line to the material. If material is not included in the article's Creative Commons licence and your intended use is not permitted by statutory regulation or exceeds the permitted use, you will need to obtain permission directly from the copyright holder. To view a copy of this licence, visit http://creativecommons. org/licenses/by/4.0/.

\section{REFERENCES}

1. Sinclair RCF, Phillips AW, Navidi M, Griffin SM, Snowden CP. Pre-operative variables including fitness associated with complications after oesophagectomy. Anaesthesia 2017;72(12):1501-7.

2. Dent B, Griffin SM, Jones R, Wahed S, Immanuel A, Hayes N. Management and outcomes of anastomotic leaks after oesophagectomy. Br J Surg 2016;103(8):1033-8.

3. Low DE, Kuppusamy MK, Alderson D, Cecconello I, Chang AC, Darling G, et al. Benchmarking complications associated with esophagectomy. Ann Surg 2019;269(2):291-8.

4. Junemann-Ramirez M, Awan MY, Khan ZM, Rahamim JS. Anastomotic leakage post-esophagogastrectomy for esophageal carcinoma: retrospective analysis of predictive factors, management and influence on longterm survival in a high volume centre. Eur J Cardiothorac Surg 2005;27(1):3-7.

5. Low DE, Alderson D, Cecconello I, Chang AC, Darling GE, D'Journo $\mathrm{XB}$, et al. International consensus on standardization of data collection for complications associated with esophagectomy: esophagectomy complications consensus group (ECCG). Ann Surg 2015;262(2):286-94.

6. Whooley BP, Law S, Alexandrou A, Murthy SC, Wong J. Critical appraisal of the significance of intrathoracic anastomotic leakage after esophagectomy for cancer. Am $J$ Surg 2001;181(3):198-203.

7. Urschel JD. Esophagogastrostomy anastomotic leaks complicating esophagectomy: a review. Am J Surg 1995;169(6):634-40.
8. Manghelli JL, Ceppa DP, Greenberg JW, Blitzer D, Hicks A, Rieger KM, Birdas TJ. Management of anastomotic leaks following esophagectomy: when to intervene? J Thorac Dis 2019;11(1):131-7.

9. Markar S, Gronnier C, Duhamel A, Mabrut JY, Bail JP, Carrere N, et al.; FREGAT (French Eso-Gastric Tumors) Working Group, FRENCH (Fédération de Recherche EN CHirurgie), and AFC (Association Française de Chirurgie). The impact of severe anastomotic leak on long-term survival and cancer recurrence after surgical resection for esophageal malignancy. Ann Surg 2015;262(6):972-80.

10. Booka E, Takeuchi H, Nishi T, Matsuda S, Kaburagi T, Fukuda $\mathrm{K}$, et al. The impact of postoperative complications on survivals after esophagectomy for esophageal cancer. Medicine (Baltimore) 2015;94(33):e1369.

11. Kataoka K, Takeuchi H, Mizusawa J, Igaki H, Ozawa S, Abe T, et al. Prognostic impact of postoperative morbidity after esophagectomy for esophageal cancer: exploratory analysis of JCOG9907. Ann Surg 2017;265(6):1152-7.

12. Martin LW, Swisher SG, Hofstetter W, Correa AM, Mehran RJ, Rice DC, et al. Intrathoracic leaks following esophagectomy are no longer associated with increased mortality. Ann Surg 2005;242(3):392-399 (discussion 399-402).

13. Phillips AW, Dent B, Navidi M, Immanuel A, Griffin SM. Trainee involvement in Ivor Lewis esophagectomy does not negatively impact outcomes. Ann Surg 2018;267(1):94-98.

14. Mapstone N. Dataset for the Histopattological Reporting of Oesophageal Carcinoma, Royal College of Pathologists. 2nd edn. 2007

15. Lagarde SM, Phillips AW, Navidi M, Disep B, Griffin SM. Clinical outcomes and benefits for staging of surgical lymph node mapping after esophagectomy. Dis Esophagus 2017;30(12):1-7.

16. Rice TW, Gress DM, Patil DT, Hofstetter WL, Kelsen DP, Blackstone EH. Cancer of the esophagus and esophagogastric junction-major changes in the American Joint Committee on Cancer eighth edition cancer staging manual. CA Cancer J Clin 2017;67(4):304-17.

17. Clavien PA, Barkun J, de Oliveira ML, Vauthey JN, Dindo D, Schulick RD, et al. The Clavien-Dindo classification of surgical complications: five-year experience. Ann Surg 2009;250(2):187-96.

18. Kamarajah SK. Adjuvant radiotherapy following pancreaticoduodenectomy for ampullary adenocarcinoma improves survival in node-positive patients: a propensity score analysis. Clin Transl Oncol 2018;20(9):1212-8.

19. Kamarajah SK, Sonnenday CJ, Cho CS, Frankel TL, Bednar F, Lawrence TS, et al. Association of adjuvant radiotherapy with survival after margin-negative resection of pancreatic ductal adenocarcinoma: a propensity-matched National Cancer Database (NCDB) Analysis. Ann Surg. 2019. https://doi.org/10.1097/sla.0000000000003242.

20. Munasinghe A, Markar SR, Mamidanna R, Darzi AW, Faiz OD, Hanna GB, et al. Is it time to centralize high-risk cancer care in the United States? comparison of outcomes of esophagectomy between England and the United States. Ann Surg 2015;262(1):79-85.

21. Brusselaers N, Mattsson F, Lagergren J. Hospital and surgeon volume in relation to long-term survival after oesophagectomy: systematic review and meta-analysis. Gut 2014;63(9):1393-400.

22. Coupland VH, Lagergren J, Luchtenborg M, Jack RH, Allum W, Holmberg L, et al. Hospital volume, proportion resected and mortality from oesophageal and gastric cancer: a populationbased study in England, 2004-2008. Gut 2013;62(7):961-6.

23. Umpleby HC, Fermor B, Symes MO, Williamson RC. Viability of exfoliated colorectal carcinoma cells. Br J Surg 1984;71(9):659-63.

24. Lindner K, Fritz M, Haane C, Senninger N, Palmes D, Hummel R. Postoperative complications do not affect long-term outcome in esophageal cancer patients. World J Surg 2014;38(10):2652-61.

25. Xia BT, Rosato EL, Chojnacki KA, Crawford AG, Weksler B, Berger AC. Major perioperative morbidity does not affect long-term survival 
in patients undergoing esophagectomy for cancer of the esophagus or gastroesophageal junction. World J Surg 2013;37(2):408-15.

26. Rutegard M, Lagergren P, Rouvelas I, Mason R, Lagergren J. Surgical complications and long-term survival after esophagectomy for cancer in a nationwide Swedish cohort study. Eur J Surg Oncol 2012;38(7):555-61.

27. Yamashita K, Makino T, Miyata H, Miyazaki Y, Takahashi T, Kurokawa $\mathrm{Y}$, et al. Postoperative infectious complications are associated with adverse oncologic outcomes in esophageal cancer patients undergoing preoperative chemotherapy. Ann Surg Oncol 2016;23(6):2106-14.

28. Baba Y, Yoshida N, Shigaki H, Iwatsuki M, Miyamoto Y, Sakamoto Y, et al. Prognostic impact of postoperative complications in 502 patients with surgically resected esophageal squamous cell carcinoma: a retrospective single-institution study. Ann Surg 2016;264(2):305-11.

29. Visser E, Markar SR, Ruurda JP, Hanna GB, van Hillegersberg R. Prognostic value of lymph node yield on overall survival in esophageal cancer patients: a systematic review and meta-analysis. Ann Surg 2019;269(2):261-8.

30. Phillips AW, Lagarde SM, Navidi M, Disep B, Griffin SM. Impact of extent of lymphadenectomy on survival, post neoadjuvant chemotherapy and transthoracic esophagectomy. Ann Surg 2017;265(4):750-6.

Publisher's Note Springer Nature remains neutral with regard to jurisdictional claims in published maps and institutional affiliations. 